\title{
Some Properties of Fractional Calculus and Linear Operators Associated with Certain Subclass of Multivalent Functions
}

\author{
Sh. Khosravianarab, ${ }^{1}$ S. R. Kulkarni, ${ }^{2}$ and O. P. Ahuja ${ }^{3}$ \\ ${ }^{1}$ Department of Mathematics, Pune University, 411007 Pune, India \\ ${ }^{2}$ Department of Mathematics, Fergusson College, 411004 Pune, India \\ ${ }^{3}$ Department of Mathematical Sciences, Kent State University, 14111 Claridon Troy Road, Burton, \\ OH 44021-9500, USA
}

Correspondence should be addressed to Sh. Khosravianarab, shkhosravian@yahoo.com

Received 27 March 2009; Accepted 25 July 2009

Recommended by Narendra Kumar Govil

We investigate several distortion inequalities involving fractional calculus, Ruscheweyh derivatives, and some well-known integral operators. In special cases, the results presented in this paper provide new approaches to several previously known results.

Copyright (c) 2009 Sh. Khosravianarab et al. This is an open access article distributed under the Creative Commons Attribution License, which permits unrestricted use, distribution, and reproduction in any medium, provided the original work is properly cited.

\section{Introduction}

Let $\mathcal{A}(p)$ denote the class of functions $f$ of the form

$$
f(z)=z^{p}+\sum_{k=1}^{\infty} a_{p+k} z^{p+k} \quad(p \in \mathbb{N}=\{1,2,3, \ldots\})
$$

which are analytic in the open unit disc $U=\{z \in \mathbb{C}:|z|<1\}$. Also, let $\mathcal{K}(p)$ denote the subclass of $\mathcal{A}(p)$ consisting of all functions $f$ of the form

$$
f(z)=z^{p}-\sum_{k=1}^{\infty} a_{p+k} z^{p+k} \quad\left(p \in \mathbb{N}=\{1,2,3, \ldots\}, a_{p+k} \geq 0\right),
$$


For functions $f, g \in \mathcal{A}(p)$, given by

$$
f(z):=z^{p}+\sum_{k=1}^{\infty} a_{p+k} z^{p+k}, \quad g(z):=z^{p}+\sum_{k=1}^{\infty} b_{p+k} z^{p+k}
$$

the Hadamard product (or convolution) of $f$ and $g$ is defined by

$$
(f * g)(z):=z^{p}+\sum_{k=1}^{\infty} a_{p+k} b_{p+k} z^{p+k}
$$

The Ruscheweyh derivative of $f$ of order $\delta+p-1$ is defined by

$$
D^{\delta+p-1} f(z):=\frac{z^{p}}{(1-z)^{\delta+p}} * f(z)=z^{p}+\sum_{k=1}^{\infty} \frac{(\delta+p)_{k}}{(1)_{k}} a_{p+k} z^{p+k}
$$

where $f$ is given by (1.1) and $\delta>-p$. The Ruscheweyh derivative $D^{\delta+p-1}$ has been studied by several authors; for example, see $[1,2]$.

For $\beta<1, \gamma \geq 0, p \in \mathbb{N}$, and $\delta>-p$, let $\tau_{\delta, p, r}(\beta)$ consist of functions $f \in \mathcal{K}(p)$ so that

$$
\mathfrak{R}\left\{e^{i \eta}\left((1-\gamma) \frac{D^{\delta+p-1} f(z)}{z^{p}}+\gamma \frac{\left(D^{\delta+p-1} f(z)\right)^{\prime}}{p z^{p-1}}-\beta\right)\right\}>0, \quad z \in \mathcal{U},
$$

for some $\eta \in \mathbb{R}$. In [3], the authors obtained four containment results for the class $\tau_{\delta, p, r}(\beta)$. We denote $\tau_{0,1, \gamma}(\beta)=\tau_{\gamma}(\beta)$. The class $\tau_{\gamma}(\beta)$ was studied by Swaminathan [4-6], Barnard et al. [7], Kim and Rønning [8], and others.

In the present paper, we investigate several distortion inequalities involving fractional calculus, Ruscheweyh derivative, and some well-known integral operators defined on the class $\tau_{\delta, p, r}(\beta)$. In special cases, the results presented here provide new approaches to some previously known results.

Remark 1.1. Throughout this section, we assume that $\delta+p \geq 1$.

\section{Definitions and Lemmas}

For the function $f$ given by (1.2), we define

$$
q_{\delta, p, \gamma}(z)=(1-\gamma) \frac{D^{\delta+p-1} f(z)}{z^{p}}+\gamma \frac{\left(D^{\delta+p-1} f(z)\right)^{\prime}}{p z^{p-1}} .
$$

It is easy to verify that

$$
q_{\delta, p, r}(z)=1-\sum_{k=1}^{\infty} \frac{(\delta+p)_{k}(p+k \gamma)}{p(1)_{k}} a_{p+k} z^{k}, \quad a_{p+k} \geq 0 .
$$


Lemma 2.1. Let the function $f$ be given by (1.2). Then $f \in \tau_{\delta, p, r}(\beta)$ if and only if

$$
\sum_{k=1}^{\infty} \frac{(\delta+p)_{k}(p+k \gamma)}{p(1)_{k}} a_{p+k} \leq \frac{1}{2}\left(\left|1+e^{i \eta}(1-\beta)\right|-\left|1-e^{i \eta}(1-\beta)\right|\right)
$$

for some $\eta \in \mathbb{R}$.

Proof. Using the fact that $\operatorname{Re}(\omega) \geq 0$ if and only if $|1+\omega| \geq|1-\omega|$, it suffices to show that

$$
\left|1+e^{i \eta}\left(q_{\delta, p, \gamma}(z)-\beta\right)\right|-\left|1-e^{i \eta}\left(q_{\delta, p, \gamma}(z)-\beta\right)\right| \geq 0,
$$

where $q_{\delta, p, r}(z)$ is defined by (2.1). Letting

$$
\mathscr{A}_{k}=\frac{(\delta+p)_{k}(p+k \gamma)}{p(1)_{k}}
$$

and assuming (2.3), we obtain

$$
\begin{aligned}
\mid 1 & +e^{i \eta}\left(q_{\delta, p, r}(z)-\beta\right)|-| 1-e^{i \eta}\left(q_{\delta, p, r}(z)-\beta\right) \mid \\
& =\left|1+e^{i \eta}(1-\beta)-e^{i \eta} \sum_{k=1}^{\infty} A_{k} a_{p+k} z^{k}\right|-\left|1-e^{i \eta}(1-\beta)+e^{i \eta} \sum_{k=1}^{\infty} A_{k} a_{p+k} z^{k}\right| \\
& \geq\left|1+e^{i \eta}(1-\beta)\right|-\left|e^{i \eta}\right|\left|\sum_{k=1}^{\infty} A_{k} a_{p+k} z^{k}\right|-\left|1+e^{i \eta}(1-\beta)\right|-\left|e^{i \eta}\right|\left|\sum_{k=1}^{\infty} A_{k} a_{p+k} z^{k}\right| \\
& \geq\left|1+e^{i \eta}(1-\beta)\right|-\left|1-e^{i \eta}(1-\beta)\right|-2 \sum_{k=1}^{\infty} A_{k} a_{p+k} \geq 0,
\end{aligned}
$$

where $z \in \partial \mathfrak{U}=\{z ; \quad z \in \mathbb{C}$ and $|z|=1\}$. By (2.3), the desired inequality (2.4) follows at once. Conversely, if $f \in \tau_{\delta, p, \gamma}(\beta)$, then

$$
\operatorname{Re}\left\{e^{i \eta}\left(q_{\delta, p, r}(z)-\beta\right)\right\}>0,
$$

or, equivalently (2.4). This yields

$$
\left|1+e^{i \eta}\left(1-\sum_{k=1}^{\infty} A_{k} a_{p+k} z^{k}-\beta\right)\right| \geq\left|1-e^{i \eta}\left(1-\sum_{k=1}^{\infty} A_{k} a_{p+k} z^{k}-\beta\right)\right|,
$$

which implies that

$$
\left|1+e^{i \eta}(1-\beta)-e^{i \eta} \sum_{k=1}^{\infty} A_{k} a_{p+k} z^{k}\right| \geq\left|1-e^{i \eta}(1-\beta)+e^{i \eta} \sum_{k=1}^{\infty} A_{k} a_{p+k} z^{k}\right| .
$$


Squaring the above inequality, choosing the value of $z$ on the half line $z=r e^{-i \theta}(0 \leq r<1)$, and letting $r \rightarrow 1^{-}$through this line, we obtain

$$
\begin{aligned}
& \left|1+e^{i \eta}(1-\beta)\right|^{2}+\left|e^{i \eta}\right|^{2}\left|\sum_{k=1}^{\infty} A_{k} a_{p+k} z^{k}\right|^{2}-2\left|1+e^{i \eta}(1-\beta)\right|\left|\sum_{k=1}^{\infty} A_{k} a_{p+k} z^{k}\right| \\
& \geq\left|1-e^{i \eta}(1-\beta)\right|^{2}+\left|e^{i \eta}\right|^{2}\left|\sum_{k=1}^{\infty} A_{k} a_{p+k} z^{k}\right|^{2}+2\left|1-e^{i \eta}(1-\beta)\right|\left|\sum_{k=1}^{\infty} A_{k} a_{p+k} z^{k}\right| .
\end{aligned}
$$

Hence we get

$$
\begin{gathered}
2\left|\sum_{k=1}^{\infty} A_{k} a_{p+k} z^{k}\right|\left(\left|1-e^{i \eta}(1-\beta)\right|+\left|1+e^{i \eta}(1-\beta)\right|\right) \leq\left|1+e^{i \eta}(1-\beta)\right|^{2}-\left|1-e^{i \eta}(1-\beta)\right|^{2} \\
=\left(\left|1+e^{i \eta}(1-\beta)\right|-\left|1-e^{i \eta}(1-\beta)\right|\right)\left(\left|1+e^{i \eta}(1-\beta)\right|+\left|1-e^{i \eta}(1-\beta)\right|\right),
\end{gathered}
$$

which reduces to

$$
2 \sum_{k=1}^{\infty} A_{k} a_{p+k} \leq\left|1+e^{i \eta}(1-\beta)\right|-\left|1-e^{i \eta}(1-\beta)\right| .
$$

So the desired inequality (2.3) follows upon using (2.5).

Setting $\eta=\delta=0$ and $p=1$ in Lemma 2.1, we get the following result.

Corollary 2.2 ([5, Theorem 2.4]). Let $f(z)$ be of the form (1.2). Then necessary and sufficient condition for $f$ to be in $\tau_{\gamma}(\beta)$ is

$$
\sum_{k=2}^{\infty}[1+\gamma(k-1)] a_{k} \leq 1-\beta, \quad a_{k} \geq 0
$$

Throughout this paper, we define

$$
E_{\eta, \beta}:=\left|1+e^{i \eta}(1-\beta)\right|-\left|1-e^{i \eta}(1-\beta)\right| .
$$

As an immediate consequence of Lemma 2.1, we have the following corollary.

Corollary 2.3. Let the function $f$ be defined by (1.2). If $f \in \tau_{\delta, p, r}(\beta)$, then

$$
a_{p+k} \leq \frac{p(1)_{k} E_{\eta, \beta}}{2(\delta+p)_{k}(p+k \gamma)}
$$

for some $\eta \in \mathbb{R}$. 
Let $\alpha_{1}, \alpha_{2}, \ldots, \alpha_{p}$ and $\beta_{1}, \beta_{2}, \ldots, \beta_{q}(p, q \in \mathbb{N} \cup\{0\}, p \leq q+1)$ be complex numbers such that $\beta_{k} \neq 0,-1,-2, \ldots$ for $k \in\{1,2, \ldots, q\}$. The generalized hypergeometric function ${ }_{p} F_{q}$ is given by

$$
{ }_{p} F_{q}\left(\alpha_{1}, \ldots, \alpha_{p} ; \beta_{1}, \ldots, \beta_{q} ; z\right):=\sum_{n=0}^{\infty} \frac{\left(\alpha_{1}\right)_{n}\left(\alpha_{2}\right)_{n} \cdots\left(\alpha_{p}\right)_{n}}{\left(\beta_{1}\right)_{n}\left(\beta_{2}\right)_{n} \cdots\left(\beta_{q}\right)_{n}} \frac{z^{n}}{n !}, \quad z \in \mathcal{U}
$$

where $(x)_{n}$ denotes the Pochhammer symbol defined by

$$
(x)_{n}=\frac{\Gamma(x+n)}{\Gamma(x)}=x(x+1)(x+2) \cdots(x+n-1), \quad \text { for } n \in \mathbb{N},(x)_{0}=1 .
$$

The operator ${ }_{p} F_{q}$ has recently been studied by several authors; for example, $[3,5]$. For $p=$ $q+1=2$, the above series give rise to the Gaussian hypergeometric series $F(a, b ; c ; z)$.

In [9], Hohlov introduced the convolution operator $H_{a, b ; c}$ by

$$
H_{a, b ; c}(f)(z):=z F(a, b ; c ; z) * f(z), \quad f \in \mathbb{A}(1) .
$$

Motivated by the operator $H_{a, b ; c}$, the authors in [3] defined the convolution operators $G_{f}^{p}$ and $H_{a, b ; c}^{p, d}$ as follows:

$$
\begin{gathered}
G_{f}^{p}(a, b ; z):=G(z):=\left(\sum_{k=1}^{\infty} \frac{(1+a)(1+b)}{(k+a)(k+b)} z^{k+p-1}\right) * f(z), \quad(a>-1, b>-1), \\
H_{a, b ; c}^{p, d}(f)(z)=H(z)=z^{p}{ }_{3} F_{2}\left(a, b, 1+\frac{p}{d^{\prime}} ; \frac{p}{d}, c ; z\right) * f(z),
\end{gathered}
$$

where $f \in \mathcal{A}(p), p \in \mathbb{N}$, and $d \geq 0$. For $p=1$, the operator $G_{f}^{1}(a, b ; z)$ was introduced in [7].

In Section 3, we will make use of the following well-known fractional calculus operators $D_{z}^{-\mu}, D_{z}^{\mu}$, and $D_{z}^{n+\mu}$. For an analytic function $f$ defined in a simply connected region of the complex $z$-plane containing the origin, these operators are defined as follows (See $[1,10])$ :

$$
D_{z}^{-\mu} f(z)=\frac{1}{\Gamma(\mu)} \int_{0}^{z} \frac{f(t)}{(z-t)^{1-\mu}} d t \quad(\mu>0)
$$

where multiplicity of $(z-t)^{\mu-1}$ is removed by requiring $\log (z-t)$ to be real when $z-t>0$;

$$
D_{z}^{\mu} f(z)=\frac{1}{\Gamma(1-\mu)} \int_{0}^{z} \frac{f(t)}{(z-t)^{\mu}} d t \quad(0 \leq \mu<1)
$$

where the multiplicity of $(z-t)^{-\mu}$ is removed, as in the definition of $D_{z}^{-\mu} f(z)$;

$$
D_{z}^{n+\mu} f(z):=\frac{d^{n}}{d z^{n}} D_{z}^{\mu} f(z) \quad\left(0 \leq \mu<1 ; n \in \mathbb{N}_{0}=\mathbb{N} \cup\{0\}\right) .
$$


By virtue of (2.21), (2.22), (2.23) and in terms of Gamma function, it is wellknown (see for details [11]) that

$$
\begin{gathered}
D_{z}^{-\mu} z^{k}=\frac{\Gamma(k+1)}{\Gamma(k+\mu+1)} z^{k+\mu} \quad(k \in \mathbb{N}, \mu>0), \\
D_{z}^{\mu} z^{k}=\frac{\Gamma(k+1)}{\Gamma(k-\mu+1)} z^{k-\mu} \quad(k \in \mathbb{N}, 0 \leq \mu<1), \\
D_{z}^{q+\mu} z^{k}=\frac{d^{q}}{d z^{q}} D_{z}^{\mu} z^{k}=\frac{\Gamma(k+1)}{\Gamma(k-q-\mu+1)} z^{k-(q+\mu),},
\end{gathered}
$$

where $q \in \mathbb{N}_{0}, k \in \mathbb{N}, 0 \leq \mu<1$, and $q \leq k$ for $\mu=0$.

In Section 4 , we will investigate the integral operator $J_{\delta, p}$ defined by

$$
\left(J_{\delta, p} f\right)(z)=\frac{\delta+p}{z^{p}} \int_{0}^{z} t^{\delta-1} f(t) d t
$$

where $f \in \mathcal{A}(p), \delta>-p$, and $p \in \mathbb{N}$. For $p=1$ and $\delta=0$, the operator was first defined by Bernardi [12]. Later on several authors studied the operator $J_{\delta, p}$; for example, see $[1,5]$.

\section{Distortion Inequalities of Convolution Operators}

Theorem 3.1. Let the function $f$ defined by (1.2) be in the class $\tau_{\delta, p, r}(\beta)$. Then

$$
\begin{aligned}
& \left|q_{\delta, p, r}(z)\right| \geq 1-\frac{1}{2} E_{\eta, \beta}|z|, \\
& \left|q_{\delta, p, r}(z)\right| \leq 1+\frac{1}{2} E_{\eta, \beta}|z|
\end{aligned}
$$

for some $\eta \in \mathbb{R}$. Here, $q_{\delta, p, r}(z)$ and $E_{\eta, \beta}$ are defined, respectively, by (2.1) and (2.14).

Proof. From (2.2), we have

$$
\begin{aligned}
\left|q_{\delta, p, r}(z)\right| & \geq 1-\left|\sum_{k=1}^{\infty} \frac{(\delta+p)_{k}(p+k \gamma)}{p(1)_{k}} a_{p+k} z^{k}\right| \\
& \geq 1-\sum_{k=1}^{\infty} \frac{(\delta+p)_{k}(p+k \gamma)}{p(1)_{k}} a_{p+k}|z|^{k} \\
& \geq 1-|z| \sum_{k=1}^{\infty} \frac{(\delta+p)_{k}(p+k \gamma)}{p(1)_{k}} a_{p+k} .
\end{aligned}
$$

Making use of Lemma 2.1, we get

$$
\left|q_{\delta, p, r}(z)\right| \geq 1-\frac{1}{2} E_{\eta, \beta}|z|
$$


for some $\eta \in \mathbb{R}$. Similarly,

$$
\left|q_{\delta, p, \gamma}(z)\right| \leq 1+|z| \sum_{k=1}^{\infty} \frac{(\delta+p)_{k}(1+(k \gamma / p))}{(1)_{k}} a_{p+k} \leq 1+\frac{1}{2} E_{\eta, \beta}|z|
$$

for some $\eta \in \mathbb{R}$. This completes the proof.

We next obtain distortion inequalities for the fractional operaters $D_{z}^{\mu}$ and $D_{z}^{-\mu}$.

Theorem 3.2. Suppose $\mu \leq(1+k+p) /(k+b+2)$ and $p<b+1$. If $f \in \tau_{\delta, p, r}(\beta)$, then for some $\eta \in \mathbb{R}$, one has

$$
\begin{gathered}
\left|D_{z}^{\mu} G(z)\right| \geq \frac{\Gamma(p+1)}{\Gamma(p-\mu+1)}|z|^{p-\mu}\left(1-\frac{p(1+a)(1+b)(1+p)}{2(2+a)(2+b)(1-\mu+p)(\delta+p)(\gamma+p)}|z| E_{\eta, \beta}\right), \\
\left|D_{z}^{\mu} G(z)\right| \leq \frac{\Gamma(p+1)}{\Gamma(p-\mu+1)}|z|^{p-\mu}\left(1+\frac{p(1+a)(1+b)(1+p)}{2(2+a)(2+b)(1-\mu+p)(\delta+p)(\gamma+p)}|z| E_{\eta, \beta}\right),
\end{gathered}
$$

where $0 \leq \mu<1, z \in \mathcal{U}, p \in \mathbb{N}$, and the operator $G(z):=G_{f}^{p}(a, b ; z)$ was defined by (2.19).

Proof. By using (2.19), we deduce that

$$
D_{z}^{\mu} G(z)=\frac{\Gamma(p+1)}{\Gamma(p-\mu+1)} z^{p-\mu}-\sum_{k=1}^{\infty} \frac{(1+a)(1+b) \Gamma(k+p+1)}{(k+a+1)(k+b+1) \Gamma(k+p-\mu+1)} a_{p+k} z^{p+k-\mu} .
$$

Then

$$
\frac{\Gamma(p-\mu+1)}{\Gamma(p+1)} z^{\mu-p} D_{z}^{\mu} G(z)=1-\sum_{k=1}^{\infty} \theta(k) a_{p+k} z^{k}
$$

where

$$
\theta(k)=\frac{(1+a)(1+b) \Gamma(k+p+1) \Gamma(p-\mu+1)}{(k+a+1)(k+b+1) \Gamma(k+p-\mu+1) \Gamma(p+1)} \quad(k, p \in \mathbb{N}, 0 \leq \mu<1) .
$$

Since $\theta(k)$ is a decreasing function of $k$, when $\mu \leq(1+k+p) /(k+b+2)$, then

$$
0<\theta(k) \leq \theta(1)=\frac{(1+a)(1+b)(1+p)}{(2+a)(2+b)(1-\mu+p)} .
$$


Also, according to Lemma 2.1 and $\delta+p \geq 1$, we have

$$
\begin{aligned}
\frac{1}{p}(\delta+p)(p+\gamma) \sum_{k=1}^{\infty} a_{p+k} & =\frac{\Gamma(\delta+p+1)(1+(\gamma / p))}{\Gamma(\delta+p)} \sum_{k=1}^{\infty} a_{p+k} \\
& \leq \sum_{k=1}^{\infty} \frac{(\delta+p)_{k}(1+(k \gamma / p))}{(1)_{k}} a_{p+k} \\
& \leq \frac{1}{2} E_{\eta, \beta}
\end{aligned}
$$

for some $\eta \in \mathbb{R}$. Then

$$
\sum_{k=1}^{\infty} a_{p+k} \leq \frac{p}{2(\delta+p)(p+\gamma)} E_{\eta, \beta}
$$

for some $\eta \in \mathbb{R}$. From (3.7) and (3.9), we obtain

$$
\frac{\Gamma(p-\mu+1)}{\Gamma(p+1)} z^{\mu-p} D_{z}^{\mu} G(z) \geq 1-\theta(1) \sum_{k=1}^{\infty} a_{p+k} z^{k}
$$

In view of (3.11), we conclude that

$$
\left|\frac{\Gamma(p-\mu+1)}{\Gamma(p+1)} z^{\mu-p} D_{z}^{\mu} G(z)\right| \geq 1-\frac{p(1+a)(1+b)(1+p)}{2(2+a)(2+b)(1-\mu+p)(\delta+p)(\gamma+p)}|z| E_{\eta, \beta}
$$

for some $\eta \in \mathbb{R}$, and

$$
\left|\frac{\Gamma(p-\mu+1)}{\Gamma(p+1)} z^{\mu-p} D_{z}^{\mu} G(z)\right| \leq 1+\frac{p(1+a)(1+b)(1+p)}{2(2+a)(2+b)(1-\mu+p)(\delta+p)(\gamma+p)}|z| E_{\eta, \beta}
$$

for some $\eta \in \mathbb{R}$, which yield (3.5).

By letting $p=1$ and $b=a-1>0$ in Theorem 3.2, we deduce the following consequence.

Corollary 3.3. If $f \in \tau_{\delta, 1, b}(\beta)$, then for $0 \leq \mu<1, z \in \mathcal{U}, \delta \geq 0$ and some $\eta \in \mathbb{R}$

$$
\begin{aligned}
& \left|D_{z}^{\mu} G(z)\right| \geq \frac{1}{\Gamma(2-\mu)}|z|^{1-\mu}\left(1-\frac{1}{(3+b)(2-\mu)(1+\delta)}|z| E_{\eta, \beta}\right), \\
& \left|D_{z}^{\mu} G(z)\right| \leq \frac{1}{\Gamma(2-\mu)}|z|^{1-\mu}\left(1+\frac{1}{(3+b)(2-\mu)(1+\delta)}|z| E_{\eta, \beta}\right) .
\end{aligned}
$$


Theorem 3.4. Let $\mu>0, z \in \mathcal{U}$ and $p \in \mathbb{N}$. If $f \in \tau_{\delta, p, r}(\beta)$, then

$$
\begin{aligned}
& \left|D_{z}^{-\mu} G(z)\right| \geq \frac{\Gamma(p+1)}{\Gamma(p+\mu+1)}|z|^{p+\mu}\left(1-\frac{p(1+a)(1+b)(1+p)}{2(2+a)(2+b)(1-\mu+p)(\delta+p)(\gamma+p)}|z| E_{\eta, \beta}\right) \\
& \left|D_{z}^{-\mu} G(z)\right| \leq \frac{\Gamma(p+1)}{\Gamma(p+\mu+1)}|z|^{p+\mu}\left(1+\frac{p(1+a)(1+b)(1+p)}{2(2+a)(2+b)(1-\mu+p)(\delta+p)(\gamma+p)}|z| E_{\eta, \beta}\right)
\end{aligned}
$$

for some $\eta \in \mathbb{R}$. The operator $G(z):=G_{f}^{p}(a, b ; z)$ was defined by (2.19).

Proof. In view of (2.19) and (2.22), we have

$$
\frac{\Gamma(p+\mu+1)}{\Gamma(p+1)} z^{-(\mu+p)} D_{z}^{-\mu} G(z)=1-\sum_{k=1}^{\infty} \tau(k) a_{p+k} z^{k}
$$

where

$$
\tau(k)=\frac{(1+a)(1+b) \Gamma(k+p+1) \Gamma(p+\mu+1)}{(k+a+1)(k+b+1) \Gamma(k+p+\mu+1) \Gamma(p+1)} .
$$

Since $\tau$ is a decreasing function of $k$, it follows that

$$
0<\tau(k) \leq \tau(1)=\frac{(1+a)(1+b)(1+p)}{(2+a)(2+b)(1+\mu+p)}
$$

By using (3.11), (3.18), and (3.20), we get

$$
\begin{gathered}
\left|\frac{\Gamma(p+\mu+1)}{\Gamma(p+1)} z^{-(\mu+p)} D_{z}^{-\mu} G(z)\right| \geq 1-\frac{p(1+a)(1+b)(1+p)}{2(2+a)(2+b)(1+\mu+p)(\delta+p)(\gamma+p)}|z| E_{\eta, \beta}, \\
\left|\frac{\Gamma(p+\mu+1)}{\Gamma(p+1)} z^{-(\mu+p)} D_{z}^{-\mu} G(z)\right| \leq 1+\frac{p(1+a)(1+b)(1+p)}{2(2+a)(2+b)(1+\mu+p)(\delta+p)(\gamma+p)}|z| E_{\eta, \beta}
\end{gathered}
$$

for some $\eta \in \mathbb{R}$. The last two inequalities yield (3.16) and (3.17), respectively. 
Letting $\delta=0, p=1$, and $a=b+1=\mu+2$ in Theorem 3.4, we get the following result.

Corollary 3.5. Let $G_{f}^{1}(\mu+2, \mu+1 ; z)$ be defined by (2.19). If $f \in \tau_{r}(\beta)$, then

$$
\begin{aligned}
& \left|D_{z}^{-\mu} G_{f}^{1}(\mu+2, \mu+1 ; z)\right| \geq \frac{1}{\Gamma(\mu+2)}|z|^{\mu+1}\left(1-\frac{1}{(\mu+4)(\gamma+1)}|z| E_{\eta, \beta}\right), \\
& \left|D_{z}^{-\mu} G_{f}^{1}(\mu+2, \mu+1 ; z)\right| \leq \frac{1}{\Gamma(\mu+2)}|z|^{\mu+1}\left(1+\frac{1}{(\mu+4)(\gamma+1)}|z| E_{\eta, \beta}\right)
\end{aligned}
$$

for some $\eta \in \mathbb{R}, \mu>0, z \in \mathcal{U}$, and $p \in \mathbb{N}$.

We next prove the distortion theorems involving fractional calculus and generalized convolution operator defined by (2.20).

Theorem 3.6. Suppose $0 \leq \mu<1, z \in \mathcal{U}, p \in \mathbb{N}$, and $\eta \in \mathbb{R}$. Also, let $a \leq 1, b \leq p-\mu+1$, and $c \geq p+1$. If $f \in \tau_{\delta, p, r}(\beta)$, then

$$
\begin{aligned}
& \left|D_{z}^{\mu} H(f)(z)\right| \geq \frac{\Gamma(p+1)}{\Gamma(p-\mu+1)}|z|^{p-\mu}\left(1-\frac{a b(p+1)}{2 c(1-\mu+p)(\delta+p)}|z| E_{\eta, \beta}\right), \\
& \left|D_{z}^{\mu} H(f)(z)\right| \leq \frac{\Gamma(p+1)}{\Gamma(p-\mu+1)}|z|^{p-\mu}\left(1+\frac{a b(p+1)}{2 c(1-\mu+p)(\delta+p)}|z| E_{\eta, \beta}\right)
\end{aligned}
$$

for some $\eta \in \mathbb{R}$. Here, the operator $H(f)(z)$ is defined by (2.20).

Proof. By making use of (2.20), we have

$$
\begin{aligned}
D_{z}^{\mu} H(f)(z) & =D_{z}^{\mu}\left\{z^{p}-\sum_{k=1}^{\infty} \frac{(a)_{k}(b)_{k}(1+(p / \gamma))_{k}}{(p / \gamma)_{k}(c)_{k}(1)_{k}} a_{p+k} z^{p+k}\right\} \\
& =\frac{\Gamma(p+1)}{\Gamma(p-\mu+1)} z^{p-\mu}-\sum_{k=1}^{\infty} \frac{(a)_{k}(b)_{k}(1+(p / \gamma))_{k} \Gamma(p+k+1)}{(p / \gamma)_{k}(c)_{k}(1)_{k} \Gamma(p+k-\mu+1)} a_{p+k} z^{p+k-\mu}
\end{aligned}
$$

It is easy to verify that

$$
1+\frac{k \gamma}{p}=\frac{(1+(p / \gamma))_{k}}{(p / \gamma)_{k}}
$$

This implies that

$$
\frac{\Gamma(p-\mu+1)}{\Gamma(p+1)} z^{\mu-p} D_{z}^{\mu} H(f)(z)=1-\sum_{k=1}^{\infty} \lambda(k)\left(1+\frac{k \gamma}{p}\right) a_{p+k} z^{k}
$$


where

$$
\lambda(k)=\frac{(a)_{k}(b)_{k} \Gamma(p+k+1) \Gamma(p-\mu+1)}{(c)_{k}(1)_{k} \Gamma(p+k-\mu+1) \Gamma(p+1)} .
$$

Since $\lambda$ is a decreasing function of $k$, when $a \leq 1, c \geq p+1$ and $b \leq p-\mu+1$, we get

$$
0<\lambda(k) \leq \lambda(1)=\frac{a b(p+1)}{c(p-\mu+1)}
$$

From Lemma 2.1 and $\delta+p \geq 1$, we obtain

$$
(\delta+p) \sum_{k=1}^{\infty}\left(1+\frac{k \gamma}{p}\right) a_{p+k} \leq \sum_{k=1}^{\infty} \frac{(\delta+p)_{k}(1+(k \gamma / p))}{(1)_{k}} a_{p+k} \leq \frac{1}{2} E_{\eta, \beta}
$$

for some $\eta \in \mathbb{R}$. It follows from (3.26) and (3.28) that

$$
\begin{aligned}
& \left|\frac{\Gamma(p-\mu+1)}{\Gamma(p+1)} z^{\mu-p} D_{z}^{\mu} H(f)(z)\right| \geq 1-\frac{a b(p+1)}{2 c(p-\mu+1)(\delta+p)}|z| E_{\eta, \beta}, \\
& \left|\frac{\Gamma(p-\mu+1)}{\Gamma(p+1)} z^{\mu-p} D_{z}^{\mu} H(f)(z)\right| \leq 1+\frac{a b(p+1)}{2 c(p-\mu+1)(\delta+p)}|z| E_{\eta, \beta}
\end{aligned}
$$

for some $\eta \in \mathbb{R}$, which yield (3.23).

We state an obvious variant of Theorem 3.6 as follows.

Corollary 3.7. Let the function $f$ defined by (1.2) be in the class $\tau_{\delta, p, r}(\beta)$. Also let $a \leq 1, b \leq p-\mu+1$, $c \geq p+1$, and $\delta+p>1$. Then

$$
\begin{aligned}
& \left|D_{z}^{\mu} H(f)(z)\right| \geq \frac{\Gamma(p+1)}{\Gamma(p-\mu+1)}|z|^{p-\mu}\left(1-\frac{1}{2}|z| E_{\eta, \beta}\right), \\
& \left|D_{z}^{\mu} H(f)(z)\right| \leq \frac{\Gamma(p+1)}{\Gamma(p-\mu+1)}|z|^{p-\mu}\left(1+\frac{1}{2}|z| E_{\eta, \beta}\right)
\end{aligned}
$$

for some $\in \in \mathbb{R}, 0 \leq \mu<1, z \in \mathcal{U}$, and $p \in \mathbb{N}$. 
The proof of Theorem 3.8 is much akin to that of Theorem 3.6, and so it is omitted here.

Theorem 3.8. Let $a \leq 1, b \leq p+\mu+1$ and $c \geq p+1$. Also, let $\mu>0, p \in \mathbb{N}$, and $z \in \mathcal{U}$. If $f \in \tau_{\delta, p, r}(\beta)$, then

$$
\begin{aligned}
& \left|D_{z}^{-\mu} H(f)(z)\right| \geq \frac{\Gamma(p+1)}{\Gamma(p+\mu+1)}|z|^{p+\mu}\left(1-\frac{a b(p+1)}{2 c(1+\mu+p)(\delta+p)}|z| E_{\eta, \beta}\right) \\
& \left|D_{z}^{-\mu} H(f)(z)\right| \leq \frac{\Gamma(p+1)}{\Gamma(p+\mu+1)}|z|^{p+\mu}\left(1+\frac{a b(p+1)}{2 c(1+\mu+p)(\delta+p)}|z| E_{\eta, \beta}\right)
\end{aligned}
$$

for some $\eta \in \mathbb{R}$.

Next we prove the following.

Theorem 3.9. Let $a \leq 1, b \leq p-\mu+1$, and $c \geq p+1$. Also, let $0 \leq \mu<1, p \in \mathbb{N}$, and $z \in \mathcal{U}$. If $f \in \tau_{\delta, p, r}(\beta)$, then

$$
\begin{aligned}
& \left|D_{z}^{\mu}\left(D^{\delta+p-1} H(f)(z)\right)\right| \geq \frac{\Gamma(p+1)}{\Gamma(p-\mu+1)}|z|^{p-\mu}\left(1-\frac{a b(p+1)}{2 c(1-\mu+p)}|z| E_{\eta, \beta}\right), \\
& \left|D_{z}^{\mu}\left(D^{\delta+p-1} H(f)(z)\right)\right| \leq \frac{\Gamma(p+1)}{\Gamma(p-\mu+1)}|z|^{p-\mu}\left(1+\frac{a b(p+1)}{2 c(1-\mu+p)}|z| E_{\eta, \beta}\right)
\end{aligned}
$$

for some $\eta \in \mathbb{R}$.

Proof. We have

$$
D^{\delta+p-1} H(f)(z)=z^{p}-\sum_{k=1}^{\infty} A_{k} z^{p+k}
$$

where

$$
A_{k}=\frac{(\delta+p)_{k}(a)_{k}(b)_{k}(1+(p / \gamma))_{k}}{(1)_{k}(p / \gamma)_{k}(c)_{k}(1)_{k}} a_{p+k}
$$

Therefore

$$
D_{z}^{\mu}\left(D^{\delta+p-1} H(f)(z)\right)=\frac{\Gamma(p+1)}{\Gamma(p-\mu+1)} z^{p-\mu}-\sum_{k=1}^{\infty} A_{k} \frac{\Gamma(p+k+1)}{\Gamma(p+k-\mu+1)} z^{p+k-\mu} .
$$


So, from (3.25), we have

$$
\frac{\Gamma(p-\mu+1)}{\Gamma(p+1)} z^{\mu-p} D_{z}^{\mu}\left(D^{\delta+p-1} H(f)(z)\right)=1-\sum_{k=1}^{\infty} \frac{(\delta+p)_{k}(p+k \gamma)}{p(1)_{k}} \lambda(k) a_{p+k} z^{k}
$$

where $\lambda(k)$ is defined by (3.27). Since $\lambda$ is a decreasing function of $k$, when $a \leq 1, b \leq p-\mu+1$ and $c \geq p+1$, then

$$
0<\lambda(k) \leq \lambda(1)=\frac{a b(p+1)}{c(p-\mu+1)} .
$$

From (3.37), (3.38), and Lemma 2.1, we find that

$$
\begin{aligned}
& \left|\frac{\Gamma(p-\mu+1)}{\Gamma(p+1)} z^{\mu-p} D_{z}^{\mu}\left(D^{\delta+p-1} H(f)(z)\right)\right| \geq 1-\frac{a b(p+1)}{2 c(1-\mu+p)}|z| E_{\eta, \beta}, \\
& \left|\frac{\Gamma(p-\mu+1)}{\Gamma(p+1)} z^{\mu-p} D_{z}^{\mu}\left(D^{\delta+p-1} H(f)(z)\right)\right| \leq 1+\frac{a b(p+1)}{2 c(1-\mu+p)}|z| E_{\eta, \beta}
\end{aligned}
$$

for some $\eta \in \mathbb{R}$. The above inequalities lead us to the desired inequalities (3.33).

The proof of the following theorem is similar to Theorem 3.9, and so it is omitted here.

Theorem 3.10. Let $a \leq 1, b \leq p+\mu+1$, and $c \geq p+1$. Also, let $\mu>0, z \in \mathcal{U}$, and $p \in \mathbb{N}$. If $f \in \tau_{\delta, p, r}(\beta)$, then

$$
\begin{aligned}
& \left|D_{z}^{-\mu}\left(D^{\delta+p-1} H(f)(z)\right)\right| \geq \frac{\Gamma(p+1)}{\Gamma(p+\mu+1)}|z|^{p+\mu}\left(1-\frac{a b(p+1)}{2 c(1+\mu+p)}|z| E_{\eta, \beta}\right), \\
& \left|D_{z}^{-\mu}\left(D^{\delta+p-1} H(f)(z)\right)\right| \leq \frac{\Gamma(p+1)}{\Gamma(p+\mu+1)}|z|^{p+\mu}\left(1+\frac{a b(p+1)}{2 c(1+\mu+p)}|z| E_{\eta, \beta}\right)
\end{aligned}
$$

for some $\eta \in \mathbb{R}$.

Upon setting $\delta=0$ and $p=1$ in Theorems 3.6, 3.8, 3.9, and 3.10, we arrive at the following result.

Corollary 3.11. Let $a \leq 1, c \geq 2, \mu>0, z \in \mathcal{U}$, and $p \in \mathbb{N}$. If $f \in \tau_{\delta, p, r}(\beta)$, then

$$
\begin{aligned}
& \left|D_{z}^{\mu} H(f)(z)\right| \geq \frac{1}{\Gamma(2-\mu)}|z|^{1-\mu}\left(1-\frac{a b}{c(2-\mu)}|z| E_{\eta, \beta}\right) \\
& \left|D_{z}^{\mu} H(f)(z)\right| \leq \frac{1}{\Gamma(2-\mu)}|z|^{1-\mu}\left(1+\frac{a b}{c(2-\mu)}|z| E_{\eta, \beta}\right)
\end{aligned}
$$


for some $\eta \in \mathbb{R}$ and $b \leq 2-\mu$. Furthermore

$$
\begin{aligned}
& \left|D_{z}^{-\mu} H(f)(z)\right| \geq \frac{1}{\Gamma(2+\mu)}|z|^{1+\mu}\left(1-\frac{a b}{c(2+\mu)}|z| E_{\eta, \beta}\right), \\
& \left|D_{z}^{-\mu} H(f)(z)\right| \leq \frac{1}{\Gamma(2+\mu)}|z|^{1+\mu}\left(1+\frac{a b}{c(2+\mu)}|z| E_{\eta, \beta}\right)
\end{aligned}
$$

for some $\eta \in \mathbb{R}$ and $b \leq 2+\mu$.

Remark 3.12. Under the hypothesis of Corollary 3.11, $D_{z}^{\mu} H(f)(z)$ and $D_{z}^{-\mu} H(f)(z)$ are included in disks with its center at origin and radii $r$ and $R$, respectively, given by

$$
\begin{aligned}
& r=\frac{1}{\Gamma(2-\mu)}\left(1+\frac{a b}{c(2-\mu)} E_{\eta, \beta}\right) \quad(0 \leq \mu<1, b \leq 2-\mu, \text { for some } \eta \in \mathcal{R}), \\
& R=\frac{1}{\Gamma(2+\mu)}\left(1+\frac{a b}{c(2+\mu)} E_{\eta, \beta}\right) \quad(\mu>0, b \leq 2+\mu, \text { for some } \eta \in \mathcal{R}) .
\end{aligned}
$$

\section{Distortion Inequalities of Integral Operator}

In this section, we obtain the distortion theorems involving the integral operator $J_{\delta, p}$ of functions in the class $\tau_{\delta, p, r}(\beta)$ and fractional calculus operator.

Theorem 4.1. Let $0 \leq \mu<1 /(\delta+p+1), z \in \mathcal{U}$, and $p \in \mathbb{N}$. If $f \in \tau_{\delta, p, r}(\beta)$, then

$$
\begin{aligned}
& \left|D_{z}^{\mu}\left(z^{-\delta}\left(J_{\delta, p} f\right)(z)\right)\right| \geq \frac{1}{\Gamma(2-\mu)}|z|^{-\mu}\left(1-\frac{p}{2(\delta+p+1)(p+\gamma)}|z| E_{\eta, \beta}\right) \\
& \left|D_{z}^{\mu}\left(z^{-\delta}\left(J_{\delta, p} f\right)(z)\right)\right| \leq \frac{1}{\Gamma(2-\mu)}|z|^{-\mu}\left(1+\frac{p}{2(\delta+p+1)(p+\gamma)}|z| E_{\eta, \beta}\right)
\end{aligned}
$$

for some $\eta \in \mathbb{R}$. The operator $\left(J_{\delta, p} f\right)(z)$ is defined by $(2.21)$.

Proof. Using the definition (2.25), for function $f \in \mathcal{K}(p)$ of the form (1.2), we have

$$
\left(J_{\delta, p} f\right)(z)=z^{\delta}-\sum_{k=1}^{\infty} \frac{\delta+p}{p+k+\delta} a_{p+k}
$$


So

$$
D_{z}^{\mu}\left(z^{-\delta}\left(J_{\delta, p} f\right)(z)\right)=\frac{1}{\Gamma(2-\mu)} z^{-\mu}-\sum_{k=1}^{\infty} \frac{(\delta+p) \Gamma(k+1)}{(\delta+p+k) \Gamma(k-\mu+1)} a_{p+k} z^{k-\mu}
$$

Therefore, we obtain

$$
\Gamma(2-\mu) z^{\mu} D_{z}^{\mu}\left(z^{-\delta}\left(J_{\delta, p} f\right)(z)\right)=1-\sum_{k=1}^{\infty} \phi(k) a_{p+k} z^{k}
$$

where

$$
\phi(k)=\frac{(\delta+p) \Gamma(k+1) \Gamma(2-\mu)}{(\delta+p+k) \Gamma(k-\mu+1)} .
$$

Since $\phi(k)$ is a decreasing function of $k$, when $\mu<1 /(\delta+p+1)$, then

$$
0<\phi(k) \leq \phi(1)=\frac{\delta+p}{\delta+p+1}
$$

By using (3.11), (4.4), and (4.6), we get

$$
\begin{gathered}
\left|\Gamma(2-\mu) z^{\mu} D_{z}^{\mu}\left(z^{-\delta}\left(J_{\delta, p} f\right)(z)\right)\right| \geq 1-\frac{p}{2(\delta+p+1)(p+\gamma)}|z| E_{\eta, \beta}, \\
\left|\Gamma(2-\mu) z^{\mu} D_{z}^{\mu}\left(z^{-\delta}\left(J_{\delta, p} f\right)(z)\right)\right| \leq 1+\frac{p}{2(\delta+p+1)(p+\gamma)}|z| E_{\eta, \beta}
\end{gathered}
$$

for some $\eta \in \mathbb{R}$, which prove the inequalities (4.1).

The proof of the following theorem is similar to Theorem 4.1, and so it is omitted here.

Theorem 4.2. Let $\mu>0, p \in \mathbb{N}$, and $z \in \mathcal{U}$. If $f \in \tau_{\delta, p, r}(\beta)$, then

$$
\begin{aligned}
& \left|D_{z}^{-\mu}\left(z^{-\delta}\left(J_{\delta, p} f\right)(z)\right)\right| \geq \frac{1}{\Gamma(\mu+1)}|z|^{\mu}\left(1-\frac{p}{2(\delta+p+1)(\mu+1)(p+\gamma)}|z| E_{\eta, \beta}\right) \\
& \left|D_{z}^{-\mu}\left(z^{-\delta}\left(J_{\delta, p} f\right)(z)\right)\right| \leq \frac{1}{\Gamma(\mu+1)}|z|^{\mu}\left(1+\frac{p}{2(\delta+p+1)(\mu+1)(p+\gamma)}|z| E_{\eta, \beta}\right)
\end{aligned}
$$

for some $\eta \in \mathbb{R}$.

\section{Acknowledgment}

The authors thank the referee for some useful suggestions for improvement of the article. 


\section{References}

[1] M. K. Aouf, H. Silverman, and H. M. Srivastava, "Some families of linear operators associated with certain subclasses of multivalent functions," Computers $\mathcal{E}$ Mathematics with Applications, vol. 55, no. 3, pp. 535-549, 2008.

[2] H. M. Srivastava, S. Owa, and O. P. Ahuja, "A new class of analytic functions associated with the Ruscheweyh derivatives," Proceedings of the Japan Academy. Series A, vol. 64, no. 1, pp. 17-20, 1988.

[3] Sh. Khosravian-Arab, S. R. Kulkarni, and J. M. Jahangiri, "Certain properties of multivalent functions associated with Ruscheweyh derivative," in Proceeding Book of the International Symposium on 2007 "Geometric Function Theory and Applications", pp. 153-160, Istanbul Kültür University Publications, 2008.

[4] A. Swaminathan, "Inclusion theorems of convolution operators associated with normalized hypergeometric functions," Journal of Computational and Applied Mathematics, vol. 197, no. 1, pp. 15-28, 2006.

[5] A. Swaminathan, "Certain sufficiency conditions on Gaussian hypergeometric functions," Journal of Inequalities in Pure and Applied Mathematics, vol. 5, no. 4, article 83, pp. 1-10, 2004.

[6] A. Swaminathan, "Sufficiency for hypergeometric transforms to be associated with conic regions," Mathematical and Computer Modelling, vol. 44, no. 3-4, pp. 276-286, 2006.

[7] R. W. Barnard, S. Naik, and S. Ponnusamy, "Univalency of weighted integral transforms of certain functions," Journal of Computational and Applied Mathematics, vol. 193, no. 2, pp. 638-651, 2006.

[8] Y. C. Kim and F. Rønning, "Integral transforms of certain subclasses of analytic functions," Journal of Mathematical Analysis and Applications, vol. 258, no. 2, pp. 466-489, 2001.

[9] Y. E. Hohlov, “Convolution operators preserving univalent functions," Ukrainskiŭ Matematicheskĭ Zhurnal, vol. 37, no. 2, pp. 220-226, 1985.

[10] T. Sekine, S. Owa, and K. Tsurumi, "Integral means of certain analytic functions for fractional calculus," Applied Mathematics and Computation, vol. 187, no. 1, pp. 425-432, 2007.

[11] H. M. Srivastava and S. Owa, Eds., Univalent Functions, Fractional Calculus, and Their Applications, Ellis Horwood Series: Mathematics and Its Applications, Ellis Horwood, Chichester, UK; John Wiley \& Sons, New York, NY, USA, 1989.

[12] P. K. Bernardi, "Convex and starlike univalent functions," Transactions of the American Mathematical Society, vol. 135, pp. 429-446, 1969. 


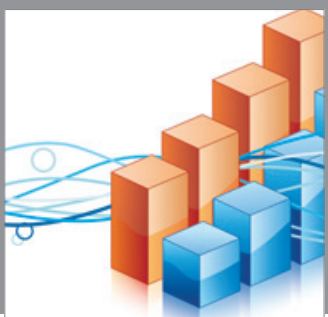

Advances in

Operations Research

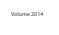

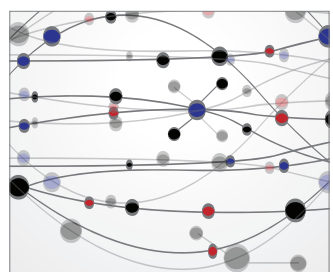

\section{The Scientific} World Journal
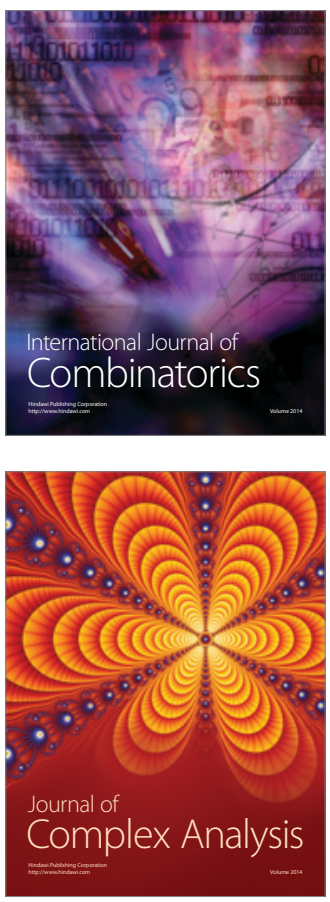

International Journal of

Mathematics and

Mathematical

Sciences
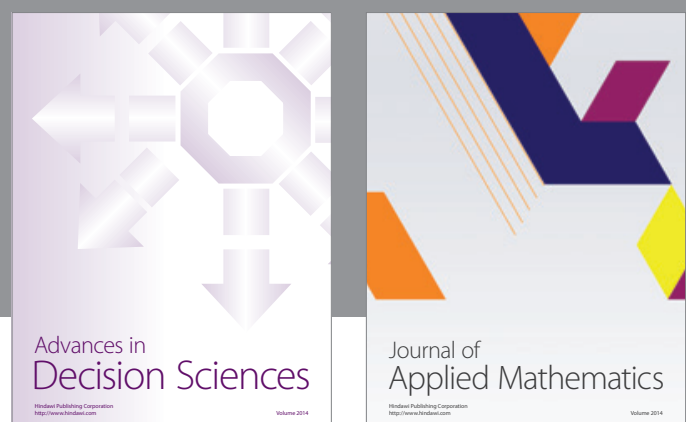

Journal of

Applied Mathematics
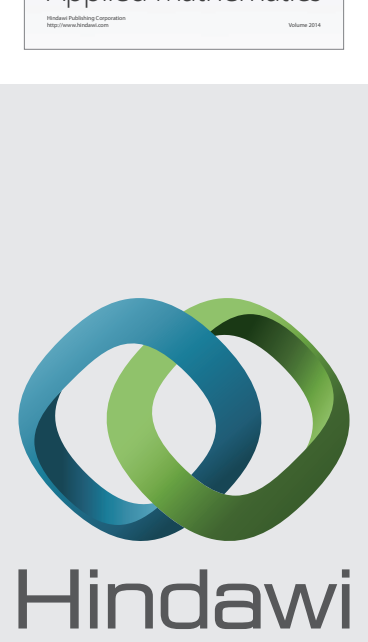

Submit your manuscripts at http://www.hindawi.com
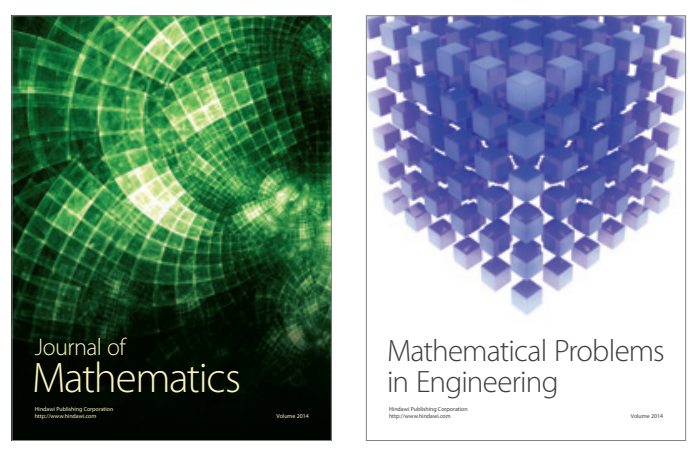

Mathematical Problems in Engineering
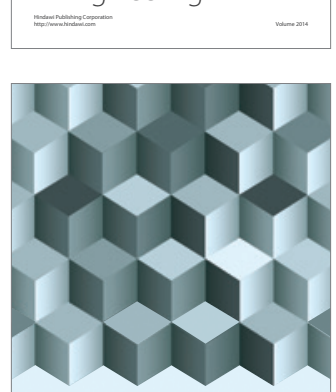

Journal of

Function Spaces
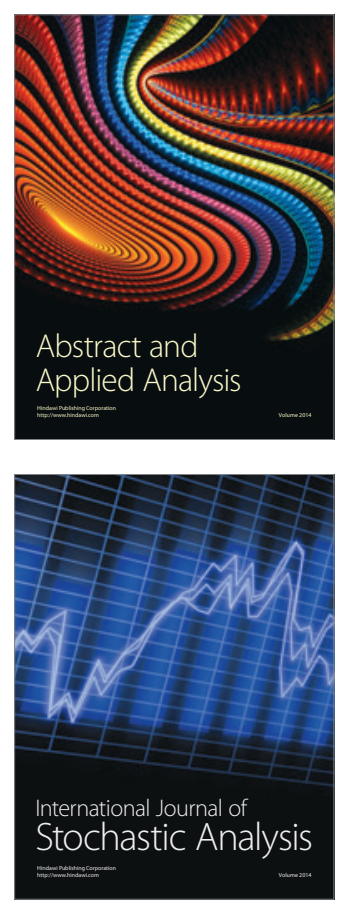

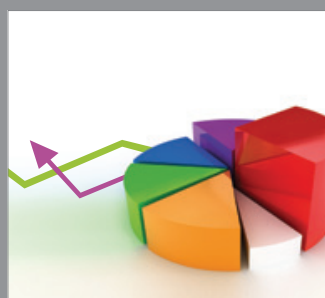

ournal of

Probability and Statistics

Promensencen
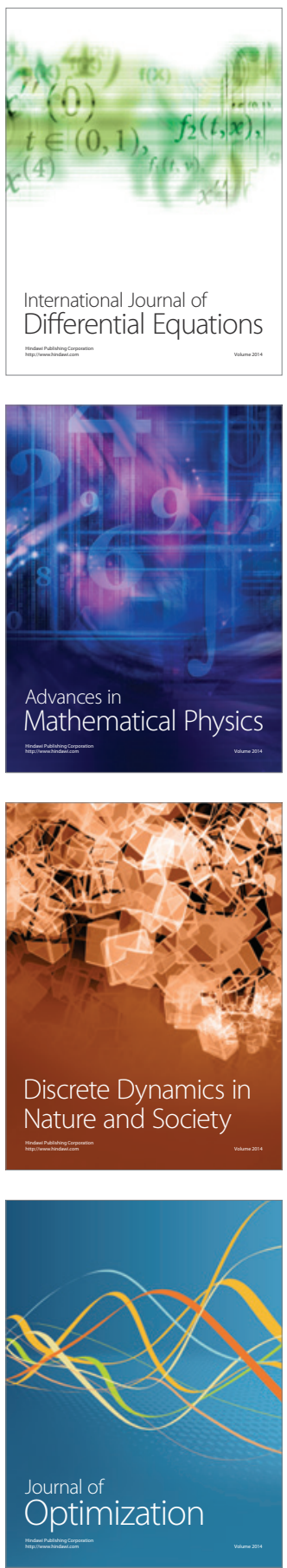